\title{
Factores que influyen en la adherencia a la vacuna contra la tosferina en mujeres embarazadas en Uruguay
}

\author{
Factors that influence the uptake of pertussis vaccine in pregnant women in Uruguay
}

\author{
Maite Inthamoussu', Stephanie Viroga ${ }^{1}$ y Noelia Speranza ${ }^{1}$
}

'Departamento de Farmacología y Terapéutica, Hospital de Clínicas Dr. Manuel Quintela, Uruguay, Facultad de Medicina, Universidad de la República. Uruguay.

Conflictos de intereses: ninguno.

Financiamiento: ninguno.

Recibido: 18 de marzo de 2021 / Aceptado: 4 de mayo de 2021

\section{Resumen}

Introducción: Desde 2015 se ofrece la vacunación contra tosferina de modo universal y gratuito a mujeres embarazadas del Uruguay. Si bien es obligatoria, la cobertura vacunal, aún no es completa. Objetivos: Conocer la prevalencia de mujeres embarazadas de dos hospitales públicos de Uruguay que recibieron vacuna dpaT en 2017 y determinar posibles factores que influyen en la adherencia a vacunarse. Métodos: Estudio observacional, transversal, descriptivo, mediante encuestas a pacientes cursando puerperio inmediato. Resultados: Se analizaron 884 encuestas (edad promedio 25,2 años; $16 \%$ adolescentes; la mayoría en pareja y educación secundaria incompleta). Se vacunaron 317 mujeres (36\%). Dentro de los factores que se asociaron a la no vacunación se destacan: adolescencia (OR 1,88; IC 95\% 1,24-2,85), no tener pareja (OR 1,40; IC 95\% 1,04-1,85), no conocer la obligatoriedad de la vacuna (OR 9,44; IC 95\% 6,63-13,45), no haber sido informada sobre los beneficios de la vacuna (OR 4; IC 95\% 2,43-6,41) y no creer en el beneficio de las vacunas en el embarazo (OR 6,37; IC 95\% 4,61-8,78). Discusión: La mayoría de las mujeres embarazadas no recibieron la vacuna dpaT ni tuvieron indicación médica. La falta de información sobre la obligatoriedad y su beneficio, y las creencias con respecto a la vacunación se asociaron a una disminución en la adherencia a la misma. Los profesionales de la salud que atienden mujeres gestantes deben recomendar e informar sobre el beneficio de la vacunación para ellas y el neonato y generar la percepción de riesgo necesaria, como una de las medidas para mejorar la cobertura vacunal.

Palabras clave: vacunación; tosferina; embarazo; vacuna acelular contra difteria, tétanos y tosferina; cobertura de vacunación; adherencia del paciente.

\section{Abstract}

Background: Since 2015, pertussis vaccine has been offered universally and free of charge to pregnant women in Uruguay. Although it is mandatory, vaccination coverage is not yet complete. Aim: To study the pertussis vaccination coverage in 2017 in pregnant women in two state hospitals and to search for barriers for uptaking the vaccine. Methods: We conducted an observational, descriptive and transversal study, using a survey in patients undergoing immediate postpartum period. Results: 884 surveys were analyzed (mean age 25.2 years; $16 \%$ teenagers, most of them in a relationship and incomplete high school). 317 women $(36 \%)$ were vaccinated. Main barriers for uptaking Tdap vaccine were: teenage and being single were associated with a greater risk for the uptake. Not being aware of the vaccine mandatoriness and not being informed about its benefits were associated with 9,44 and 4 higher risks for not uptaking the vaccine (IC 95\% 6.63-13.45 and IC $95 \% 2.43-6.41$, respectively). Not believing in the benefits of pertussis vaccine during pregnancy was associated with 6.37 higher risk (OR 6.37; IC 95\% 4.61-8.78). Discussion: Most pregnant women in this study during 2017 did not uptake pertussis vaccine and did not have medical indication for it. The lack of information about the obligation and benefits, and also patients' beliefs about the vaccination were identified as barriers. Health professionals who treat pregnant women should recommend and inform about the benefits of pertussis vaccine for women and the infant, and create the necessary perception of risk, in order to improve the vaccination coverage.

Keywords: vaccination; whooping cough; pregnancy; diphtheriatetanus-acellular pertussis vaccines; vaccination coverage; patient adherence.

\section{Correspondencia a:}

Maite Inthamoussu

maite.intha.fer@gmail.com 


\section{Introducción}

$\mathrm{L}$ a tos convulsa, o más comúnmente llamada tosferina, es una enfermedad respiratoria aguda muy contagiosa, producida por Bordetella pertussis. Se considera una enfermedad grave que se transmite de persona a persona, por gotitas de secreciones respiratorias expulsadas por la tos y clínicamente tiene un período de incubación que suele durar entre 7 y 10 días ${ }^{1}$.

La vacuna se comenzó a administrar en América en la década de los 40, con impacto en la morbimortalidad, pero aún persisten los brotes, casos graves y mortales con especial incidencia en países en desarrollo ${ }^{2,3}$.

En Uruguay, la vacuna contra la tosferina fue implementada en el año 1963. Forma parte del Programa Nacional de Vacunaciones (PNV) como vacuna pentavalente (difteria, pertussis, tétanos, hepatitis B, Haemophilus influenzae tipo b) ofrecida actualmente a los 2, 4, 6 y 15 meses de edad y como triple bacteriana celular (difteria, pertussis, tétanos) a los 5 años. En el año 2012 se incorporó la vacunación antipertussis acelular a los 12 años (como vacuna triple bacteriana acelular) y en 2015 en las mujeres gestantes, en cada embarazo. También se incorporó una estrategia de vacunación "en capullo" para proteger a los lactantes bajo seis meses de edad, que al nacer pesaron menos de $1.500 \mathrm{~g}$ y vacunación del personal de salud que esté en contacto con niños bajo 12 meses de edad. El PNV es un programa universal, gratuito y obligatorio para todos los residentes del país ${ }^{4,5}$.

Las últimas incorporaciones de vacunación antipertussis al PNV respondieron al último brote registrado en el país. En el año 2011 se registró un brote de tosferina con un total de 630 casos notificados, siendo más de $70 \%$ de los casos en edades iguales o inferiores a un año, cifra que representa el valor de casos más altos desde el año $1987^{6}$.

Un estudio observacional descriptivo, registró 34 ingresos de casos de tosferina en la Unidad de Cuidados Intensivos de Niños (UCIN) del Hospital Pediátrico del Centro Hospitalario Pereira Rossell (HP-CHPR), centro nacional de referencia pediátrica, en el período de abril a diciembre de 2011. De ellos fallecieron 9. La mediana de la edad fue de 2 meses (rango: 15 días - 60 meses), 24 pacientes tenían menos de 4 meses de edad ${ }^{7}$.

En el año 2012 se hospitalizaron en el HP-CHPR 164 niños con diagnostico probable de tosferina, de los cuales se confirmó la infección en 84 de ellos. El 50\% de los casos fueron lactantes bajo 4 meses de edad. Diecinueve niños deberían haber recibido el esquema completo de vacunación antipertussis pero sólo lo habían recibido 8 (42,1\%). Veintiuno de los 84 niños hospitalizados presentaron complicaciones. El promedio de hospitalización fue de 7 días (rango 1 a 25 días). Un 21,4\% de los niños fueron derivados a UCIN. Se registró un fallecimiento, correspondiente a un lactante de 2 meses de edad ${ }^{8}$.
Como se comentó previamente, el Ministerio de Salud Pública (MSP) ofrece desde el año 2015 la vacunación gratuita y universal de todas las mujeres embarazadas, con una vacuna triple bacteriana con componente antipertussis acelular $(\mathrm{dpaT})^{5}$.

La vacunación anti-pertussis en el embarazo es una de las estrategias más efectivas para disminuir la morbimortalidad de la tosferina en los niños bajo 6 meses de edad. Esto se logra gracias al pasaje de anticuerpos maternos al feto otorgándole protección inmunitaria mientras el niño completa su esquema primario de vacunación ${ }^{9-11}$.

El recién nacido, tiene un sistema inmune inmaduro por lo que los anticuerpos maternos clase $\operatorname{IgG}$ son su única defensa frente a la exposición a este microorganismo. El pasaje placentario de los mismos se da en el tercer trimestre del embarazo ${ }^{12,13}$.

La vacunación contra la tosferina logra disminuir la incidencia de casos de esta enfermedad infecciosa en estos niños, así como la gravedad de dicha enfermedad frente al contagio. Algunos estudios hablan de hasta $85 \%$ menos de infecciones respecto a las madres no vacunadas y una reducción entre 58 y $72 \%$ del ingreso hospitalario y asistencia ventilatoria mecánica ${ }^{13}$.

En cuanto al perfil de seguridad de esta vacuna en el embarazo, un estudio retrospectivo con más de 120.000 mujeres gestantes no encontró asociación con complicaciones obstétricas neonatales ${ }^{14}$. También se realizó un ensayo clínico randomizado para valorar, tanto los efectos adversos como el desarrollo de la enfermedad en los neonatos, no encontrándose diferencias significativas ${ }^{15}$.

Debido a que en los lactantes se observa mayor incidencia de esta enfermedad y teniendo en cuenta que los dos primeros meses de vida no se recomienda vacunar a los recién nacidos, se implementó desde el año 2015 en nuestro país, la vacunación de carácter obligatorio y gratuito a las mujeres embarazadas en todos los vacunatorios públicos y privados del país. La pauta nacional indica la vacunación entre las 28 y 36 semanas de gestación, con el objetivo de proteger al futuro recién nacido, población más vulnerable y con mayor riesgo. $\mathrm{Si}$ bien la vacunación en dicha población es obligatoria, la cobertura vacunal ha sido baja (años 2018 y 2019: 49 y $56 \%$, respectivamente) ${ }^{16}$.

Un estudio de costo-efectividad realizado en Estados Unidos de América (E.U.A.) encontró que la vacunación preparto contra dpaT es costo-efectiva, siendo esta estrategia más eficiente para la protección del neonato en sus dos primeros meses de vida, comparada con la vacunación post-parto, vacunación pre-parto de la pareja u otros miembros del grupo familiar ${ }^{10}$.

Los estudios cualitativos en mujeres gestantes facilitan la comprensión del contexto en el que transcurren los embarazos y conocer las necesidades, conocimientos y apreciaciones de las mujeres. Son un pilar fundamental 
para definir políticas de salud que permitan, por ejemplo, derribar barreras para la adherencia vacunal ${ }^{17}$.

Los objetivos del presente trabajo fueron conocer la prevalencia de las mujeres embarazadas que recibieron la vacuna contra la tosferina entre las usuarias del CHPR y maternidad del Hospital de Clínicas (HC) y determinar posibles factores de riesgo que influyen en la adherencia a la vacunación en estas mujeres.

\section{Pacientes y Métodos}

Se realizó un estudio observacional, descriptivo, de corte transversal, durante el 2017 en las maternidades de dos hospitales del subsector público de salud de Uruguay, de carácter universitario (CHPR y HC), que asisten mujeres embarazadas de todo el país. Se incluyeron pacientes que cursaban el puerperio inmediato (primeras $72 \mathrm{~h}$ post-nacimiento), de un recién nacido vivo, cuyo embarazo hubiera finalizado más allá de las 32 semanas de edad gestacional por fecha de última menstruación, que consintieron participar del estudio. Los datos se obtuvieron mediante una encuesta prediseñada por los autores.

Las variables a analizar fueron la prevalencia de pacientes vacunadas en relación al número de pacientes encuestadas y los posibles factores que intervienen en la adherencia. Dentro de estos últimos se analizaron la edad categorizando en adolescentes (menor o igual a 19 años)

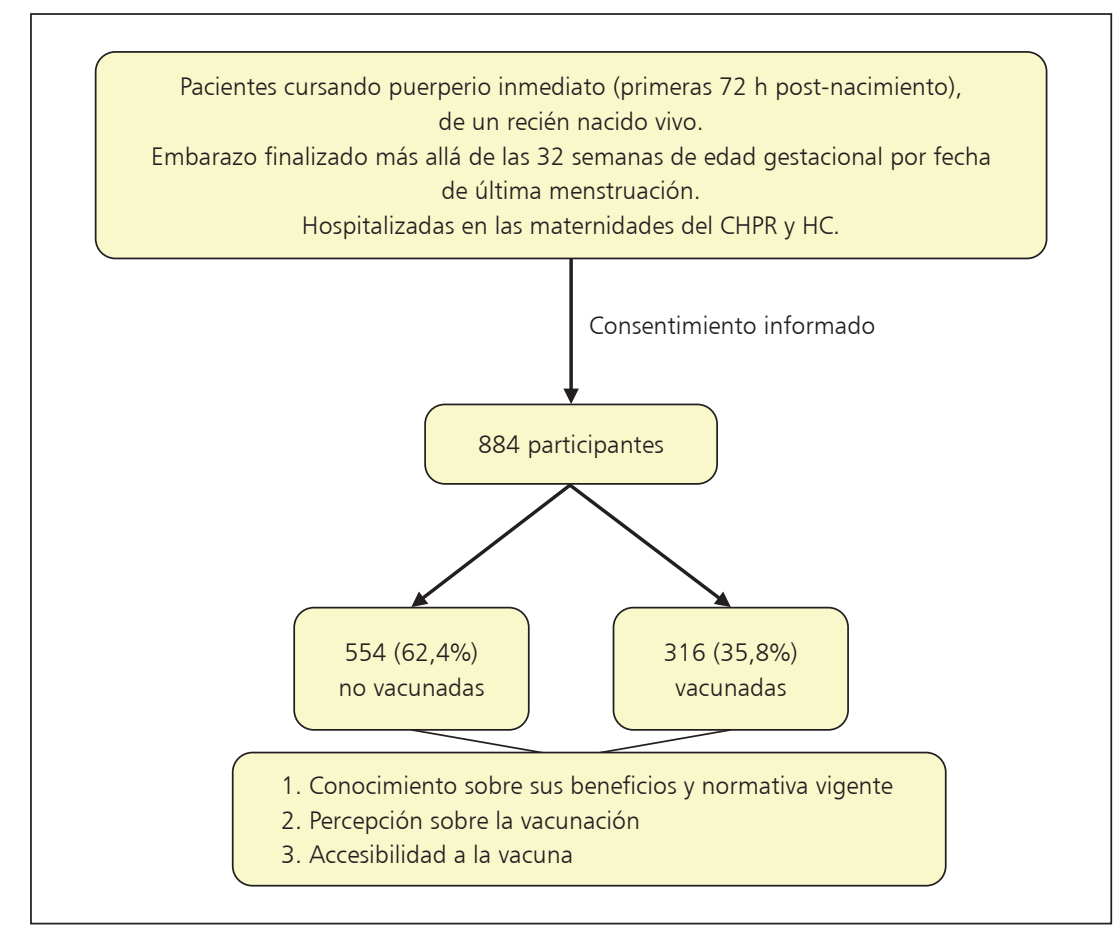

Figura 1. Flujograma de estudio. y no adolescentes (mayor a 19 años), nivel educativo (educación primaria completa/incompleta, secundaria completa/incompleta, terciaria completa/incompleta), estado civil (sola o en pareja), paridad (primigesta o multípara) y tres grandes aéreas en relación a la vacuna: conocimiento sobre sus beneficios y la normativa vigente (conocimiento sobre vacunación, obligatoriedad y beneficios), percepción sobre la vacunación (miedo a agujas, miedo a vacunación durante el embarazo, creencia en beneficios de vacunación durante el embarazo) y accesibilidad a la vacuna (vacunatorio en barrio y centro de salud).

Se calculó una muestra para la prevalencia de 382 pacientes considerando una cobertura vacunal de 50\% y un error alfa de $5 \%$. Para la determinación de factores que infieren en la adherencia se estableció una muestra de conveniencia del doble de la prevalencia $(n=764)$.

Los datos se analizaron utilizando el programa estadístico STATA $16^{\circledR}$. Para las variables continuas se utilizaron frecuencia absoluta, relativa y rango, y para las discretas media, mediana y rango. Se realizó regresión logística simple y múltiple para el análisis de los posibles factores determinantes de la no adherencia a la vacunación, y se calculó su significancia estadística (p 0,05).

El trabajo se realizó en el marco de la curricularización de la investigación en la enseñanza de pregrado, por lo que las estudiantes de la Escuela de Parteras de $3^{\circ}$ año participaron en la aplicación de las encuestas. Previo a la inclusión de pacientes se realizó entrenamiento de las estudiantes sobre la aplicación de la encuesta, con la creación de un manual para el llenado de las mismas.

Las pacientes a incluir se obtuvieron diariamente a partir del libro de nacimientos de cada maternidad. Se realizó control de calidad sobre la aplicación de las encuestas, mediante encuestadores externos.

Se obtuvo la aprobación del protocolo de los Comité de Ética de ambas instituciones de salud (HC y CHPR).

\section{Resultados}

Se realizaron 884 encuestas (Figura 1). La edad promedio fue de 25 años (14-44 años). Un 15,6\% eran adolescentes, de las cuales $25 \%$ se vacunó. El resto correspondieron a no adolescentes, de las cuales $38 \%$ se vacunó. La mayoría de las entrevistadas tenían enseñanza secundaria incompleta como máximo nivel educativo. La mayoría de las entrevistadas se encontraba al momento en pareja y eran multíparas (Tabla 1).

Del análisis descriptivo de las variables incluidas se desprende que $75,7 \%$ no conocía la vacuna ni sus beneficios $(83,1 \%)$ y la mayoría no conocía o no sabía sobre sus riesgos. A su vez, 28,9\% experimentó miedo a la vacunación durante el embarazo y $28,9 \%$ a las agujas. Por último, 79,8\% de las participantes creía en los beneficios 
de la vacunación durante el embarazo. La mayoría $(90 \%$ aproximadamente) no presentó problemas de acceso a los vacunatorios barriales o de su centro de salud (Tabla 2).

Del total de las entrevistadas la mayoría $(62,4 \%)$ no recibió vacunación contra tosferina, y $35,8 \%$ si, el resto $(1,8 \%)$ desconoce. En la mayoría $(67,6 \%)$ no había registro de vacunación en el carnet obstétrico.

Un $58,1 \%$ no tuvo indicación por parte de los profesionales de salud de vacunación (Tabla 2). De 376 participantes que recordaban al prescriptor de la vacunación (el resto de las participantes no lo recordaba), la mayoría fueron parteras $(52,4 \%)$, seguido por ginecólogo $(38,3 \%)$ y médico de familia $(4,8 \%)$.

En el análisis de los factores de riesgo que influyeron en la adherencia a la vacunación se destacó que la adolescencia y no tener pareja se asociaron con un mayor riesgo de no vacunarse (OR 1,88; IC 95\% 1,24-2,85 y OR 1,40 ; IC $95 \%$ 1,04-1,85, respectivamente) (Tabla 3). Las mujeres embarazadas que no conocían la obligatoriedad de la vacuna tuvieron un riesgo 9,44 veces mayor de no vacunarse (IC 95\% 6.63-13.45) y aquellas que no fueron informadas sobre los beneficios de la vacuna tuvieron un riesgo cuatro veces mayor (IC 95\% 2,43-6,41). No creer en el beneficio de las vacunas en el embarazo se asoció con un riesgo 6,37 veces mayor de no vacunarse (OR 6,37; IC 95\% 4,61-8,78). El resto de las variables no arrojaron resultados estadísticamente significativos (Tabla 4).

\begin{tabular}{|c|c|c|c|}
\hline & $\begin{array}{c}\text { Sí } \\
\text { (n (\%)) }\end{array}$ & $\begin{array}{c}\text { No } \\
\text { (n (\%)) }\end{array}$ & $\begin{array}{l}\text { No sabe } \\
\text { (n (\%)) }\end{array}$ \\
\hline \multicolumn{4}{|l|}{ Conocimiento de la vacuna } \\
\hline Conocimiento sobre dpaT & $215(24,3)$ & $669(75,7)$ & \\
\hline Conocimiento sobre obligatoriedad & $388(44,5)$ & $485(55,5)$ & \\
\hline Conocimiento de beneficios & $148(16,9)$ & $729(83,1)$ & $0(0,0)$ \\
\hline Indicación de vacunación & $359(40,7)$ & $513(58,1)$ & $11(1,3)$ \\
\hline \multicolumn{4}{|l|}{ Percepción de la vacuna } \\
\hline Miedo a agujas & $255(28,9)$ & $624(70,7)$ & $4(0,4)$ \\
\hline Miedo a vacunación durante el embarazo & $186(21,0)$ & $669(75,8)$ & $28(3,2)$ \\
\hline Riesgos & $80(9,1)$ & $421(47,7)$ & $382(43,2)$ \\
\hline Creencia en beneficios de vacunación durante el embarazo & $705(79,8)$ & $85(9,6)$ & $94(10,6)$ \\
\hline \multicolumn{4}{|l|}{ Accesibilidad } \\
\hline Vacunatorio en su centro de salud & $814(92,1)$ & $59(6,7)$ & $11(1,2)$ \\
\hline Vacunatorio en su barrio & $786(88,9)$ & $85(9,6)$ & $13(1,5)$ \\
\hline
\end{tabular}




\begin{tabular}{|c|c|c|c|c|c|}
\hline & Vacunación & No vacunación & Total & OR (IC 95\%) & p \\
\hline \multicolumn{6}{|l|}{ Edad } \\
\hline No adolescente & $283(38,6 \%)$ & $450(61,4 \%)$ & 733 & & \\
\hline Adolescente & $34(25 \%)$ & $102(75 \%)$ & 136 & $1,88(1,24-2,85)$ & 0,003 \\
\hline \multicolumn{6}{|l|}{ Estado civil } \\
\hline En pareja & $96(31,6 \%)$ & $208(68,4 \%)$ & 304 & & \\
\hline Sola & $221(40,5 \%)$ & $343(59,5 \%)$ & 546 & $1,40(1,04-1,87)$ & 0,02 \\
\hline \multicolumn{6}{|l|}{ Educación } \\
\hline Primaria incompleta & $15(32,6 \%)$ & $31(67,4 \%)$ & 46 & & \\
\hline Primaria completa & $89(34 \%)$ & $173(66 \%)$ & 262 & $0,94(0,48-1,83)$ & 0,86 \\
\hline Secundaria incompleta & $168(36,5 \%)$ & $292(63,5 \%)$ & 460 & $0,84(0,44-1,60)$ & 0,69 \\
\hline Secundaria completa & $34(45,3 \%)$ & $41(54,7 \%)$ & 75 & $0,58(0,27-1,25)$ & 0,17 \\
\hline Terciaria incompleta & $9(47,4 \%)$ & $10(52,6 \%)$ & 19 & $0,53(0,18-1,60)$ & 0,26 \\
\hline Terciaria completa & $2(28,6 \%)$ & $5(71,4 \%)$ & 7 & $1,21(0,21-6,97)$ & 0,83 \\
\hline \multicolumn{6}{|l|}{ Paridad } \\
\hline Primigesta & $101(36,3 \%)$ & $178(63,8 \%)$ & 279 & & \\
\hline Multípara & $216(36,6 \%)$ & $374(63,4 \%)$ & 590 & $0,98(0,73-1,92)$ & 0,91 \\
\hline
\end{tabular}

Tabla 4. Estudio de adherencia a la vacuna antipertussis en el embarazo. Uruguay, 2017. Factores que influyen en la adherencia a la vacunación

\begin{tabular}{|c|c|c|c|}
\hline Factores asociados a la adherencia & OR & IC $95 \%$ & $\mathbf{p}$ \\
\hline \multicolumn{4}{|l|}{ Conocimiento sobre beneficios y normativa vigente } \\
\hline Conocimiento sobre dpaT & 1,0 & $0,7-1,6$ & 0,7 \\
\hline Conocimiento sobre obligatoriedad & 9,4 & $6,6-13,4$ & 0,0 \\
\hline Conocimiento de beneficios & 3,9 & $2,4-6,4$ & 0,0 \\
\hline \multicolumn{4}{|l|}{ Percepción sobre vacunación } \\
\hline Miedo a agujas & 0,8 & $0,5-1,1$ & 0,2 \\
\hline Miedo a vacunas en el embarazo & 1,4 & $0,9-2,1$ & 0,1 \\
\hline No creencia en beneficio durante el embarazo & 6,3 & $4,6-8,7$ & 0,0 \\
\hline \multicolumn{4}{|l|}{ Accesibilidad a vacunas } \\
\hline Carencia de vacunatorio en centro de salud & 1,2 & $0,7-2,2$ & 0,4 \\
\hline Carencia de vacunatorio en el barrio & 1,0 & $0,6-1,7$ & 0,7 \\
\hline
\end{tabular}

\section{Discusión}

Según la bibliografía nacional disponible consultada, el presente trabajo es el primero realizado en nuestro país con el objetivo de conocer los factores que influyen en la vacunación contra la tosferina en el embarazo. El número de encuestadas superó el de la muestra calculada, lo que brinda aún más valor a los datos hallados.

El porcentaje de mujeres gestantes vacunadas fue bajo y menor al estimado a nivel nacional para los años 2018 y 2019 (49 y 56\%, respectivamente) $)^{16}$. Otros estudios mostraron tasas algo superiores; un trabajo inglés del año 2018 tuvo una tasa de $68,8 \%$ y otro norteamericano del mismo año fue de $54,4 \%{ }^{18-20}$.

La vacunación antipertussis en el embarazo es una de las estrategias más efectivas para disminuir la morbimortalidad en los niños bajo 6 meses de edad ya que la mayoría de los casos de tosferina y las muertes por esta infección ocurren en niños antes de dos meses de edad, donde no es recomendable la vacunación y el origen de la infección, en alrededor de $35 \%$ de los casos, es intrafamiliar ${ }^{10}$.

Previo al análisis de los factores de riesgo de no adherencia se destaca que un alto porcentaje de mujeres gestantes no recibió indicación de vacunación. Este hecho puede contribuir a la no vacunación.

En relación a los posibles factores de riesgo analizados en este trabajo, la adolescencia y no tener pareja fueron determinantes en la adherencia. Cabe destacar que el nivel de instrucción no se asoció significativamente con la adherencia a la vacunación, así como tampoco la paridad de las participantes. 
de las participantes siente que los profesionales de la salud no dedican suficiente tiempo discutiendo los beneficios y riesgos de la vacunación, teniendo las parteras un potencial para influenciar positivamente la misma. A su vez, varias participantes no vacunadas expresaron que hubieran adherido a la vacunación de haber sido recomendada por los profesionales ${ }^{24}$.

En el estudio previamente comentado de Krishnaswamy y cols., entre los profesionales de la salud, $25 \%$ se consideraban poco confiados en discutir la vacuna, identificándose los obstetras significativamente más confiados que las parteras (68 vs. 55\% muy o moderadamente confiados OR 2,05, IC 95\% 1,02-4,12) ${ }^{23}$.

De estos hallazgos se destaca la necesidad de mejorar la consejería por parte del personal de salud y la existencia de campañas masivas de información pública, en centros de salud, medios de comunicación y redes sociales, entre otros. Cabe destacar que las encuestas se realizaron en centros de salud universitarios. Sería interesante conocer el comportamiento de profesionales de centros de salud fuera del ámbito universitario.

Podría actuar como factor favorecedor que en el carnet obstétrico se incluyera un ítem sobre si la mujer gestante recibió o no la vacuna dpaT. En nuestro país se ha discutido su implementación como parte de los cambios en el Sistema Informático Perinatal (sistema único de registro de los controles de las mujeres embarazadas de todo el país), lo que podría actuar como un recordatorio fácil de implementar y de alto impacto en las coberturas vacunales.

Es necesario la formación de los profesionales de la salud en la escucha atenta y capacidad de dar respuesta a las creencias y dudas respecto de la vacunación y sus riesgos, en este caso para la mujer embarazada y el feto.

En esta línea, una revisión sistemática realizada por Mohammed y cols., en el año 2019 incluyó estudios que reportaron intervenciones para mejorar la vacunación antipertussis en el embarazo. Dentro de estos se destaca un estudio retrospectivo el cual evaluó una alerta dentro del sistema electrónico médico de E.U.A., que recordaba a los profesionales de la salud ofrecer la vacunación contra la tosferina en mujeres embarazadas. Como hallazgo, esta alerta aumentó significativamente la cobertura vacunal de 48 a 97\%. Otra de las medidas destacadas fue la administración de la vacunación por parte de las parteras en los centros de salud ${ }^{25}$. En relación a esto último, en nuestro país, la vacunación es un acto llevado a cabo exclusivamente por vacunadores en centros de salud habilitados para ello.

Como limitantes de este trabajo se destaca que la muestra, aunque amplia, no incluyó a mujeres embarazadas usuarios de prestadores privados de salud ni centros de salud no universitarios. Tampoco se hizo un análisis exhaustivo de todos los posibles factores de riesgo, por 
ejemplo, el estrato socioeconómico, creencia religiosa, influencia familiar, adherencia a otras vacunas, entre otros. Por último, como toda encuesta sobre una situación pasada, existe el riesgo de sesgo de memoria entre las encuestadas.

\section{Conclusiones}

La mayoría de las mujeres embarazadas incluidas no recibieron la vacuna contra dpaT ni recibieron indicación para la misma. Se identificaron como factores de riesgo para la no vacunación ser adolescente y no estar en pareja. La falta de información sobre la obligatoriedad y el beneficio de la vacunación fueron determinantes de la no vacunación. Así mismo, las creencias con respecto a la vacunación se asociaron a una disminución en la adherencia a la misma. Los profesionales de la salud que atienden mujeres embarazadas deben recomendar e informar sobre el beneficio de la vacunación contra tos ferina, como una de las medidas para mejorar la cobertura vacunal y el beneficio esperado por la vacunación.

Agradecimientos. El presente trabajo se encuentra enmarcado en la curricularización de la investigación en la enseñanza de pregrado, por lo que estudiantes de la Escuela de Parteras, Facultad de Medicina, UdelaR, de tercer año participaron en la aplicación de la encuesta.

\section{Referencias bibliográficas}

1.- American Academy of Pediatrics. Pertussis (Whooping cough) In Kimberly DW, Brady MT, Jackson MA, Long SS, eds. Red Book 2018; Report of the. Committee on Infectious Diseases. $31^{\circ}$ ed. Itasca, Il. American Academy of Pediatrics. 2018; 620-34

2.- Bottero D, Gabriell M, Aispuro P, Regidor V, Hozbor D. Pertussis, historia, hechos y situación actual. Acta Bioquím Clín Latinoam. 2019; 53 (3): 343-52. https://www.redalyc.org/ jatsRepo/535/53562084014/53562084014.pdf.

3.- Ulloa R, Hernández M, Ávila M L. Bordetella pertussis en Latinoamérica: ¿estamos reconociendo el problema? An Pediatr (Barc). 2008; 69 (3): 197-9. doi: 10.1157/13125810.

4.- Ministerio de Salud Pública. Medidas de profilaxis contra la tos convulsa. Enero 2016. Disponible en: https:/www.asse.com.uy/ aucdocumento.aspx?8440,50204.

5.- Ministerio de Salud Pública. Informes. Vacunas. Nov 2020, actualización abril 2021. Disponible en: https:/www.gub.uy/ministeriosalud-publica/comunicacion/publicaciones/ vacunas.

6.- Ministerio de Salud Pública. Dirección General de la Salud. División de Epidemiología. Informe de actualización situación tos convulsa. Set 2012. Disponible en: https:// www.gub.uy/ministerio-salud-publica/sites/ ministerio-salud-publica/files/documentos/ publicaciones/Informe tos convulsa SETIEMBRE 2012\% $\overline{2} 0 \% \overline{2} 84 \% 29$.pdf.

7.- Serra A, Machín C, Gutiérrez C, Menchaca A. Tos convulsa: enfermedad reemergente. Arch. Pediatr. Urug. 2013 Dic; 84 (4): 255-62. http:// www.scielo.edu.uy/pdf/adp/v84n4/v84n4a02. pdf.

8.- Sosa M, Castro M, Salomón S, Giachetto G. Características epidemiológicas y clínicas de niños hospitalizados con tos convulsa durante el 2021 en el Hospital Pediátrico del Centro Hospitalario Pereira Rossell. Arch Pediatr Urug. 2021; 85 (1): 10-17. http://www.scielo. edu.uy/pdf/adp/v85n1/v85n1a03.pdf.

9.- $\quad$ Sawyer M, Liang J L, Messonnier N, Clark T. Updated recommendations for use of tetanus toxoid, reduced diphtheria toxoid, and acellular pertussis vaccine (Tdap) in pregnant women--Advisory Committee on Immunization Practices (ACIP), 2012. MMWR Morb Mortal Wkly Rep. 2013; 62 (7): 131-5. https://www. cdc.gov/mmwr/preview/mmwrhtml/mm6207a4. htm.

10.- Atkins K, Fitzpatrick M, Galvani A, Towsend J. Cost-effectiveness of pertussis vaccination during pregnancy in the United States. Am J Epidemiol. 2016; 183 (12): 1159-70. doi: 10.1093/aje/kwv347.

11.- Vizzotti C, Neyro S, Katz N, Juárez M V, Pérez Carrega M E, Aquino A, et al. Maternal immunization in Argentina: A storyline from the prospective of a middle income country. Vaccine; 2015; 33 (47): 6413-9. doi: 10.1016/j. vaccine.2015.07.109

12.- Torres L S, Gamboa D Á. Inmunología perinatal. FEMINA. 2014; 42 (4): 185-92. http://files.bvs.br/upload/S/0100-7254/2014/ v42n4/a4591.pdf.

13.- Winter K, Nickell S, Powell M, Harriman K Effectiveness of prenatal versus postpartum tetanus, diphtheria, and acellular pertussis vaccination in preventing infant pertussis. Clin Infect Dis. 2017; 64: 3-8. doi: 10.1093/cid/ ciw634.

14.- Kharbanda E O, Vázquez-Benítez G, Lipkind H S, Klein N P, Cheetham T C, Naleway A, et al. Evaluation of the association of maternal pertussis vaccination with obstetric events and birth outcomes JAMA. 2014; 312 (18): 1897-904. doi: 10.1001/jama.2014.14825.

15.- Munoz F M, Bond N H, Maccato M, Pinell
P, Hammill H A, Swamy G K, et al. Safety and immunogenicity of tetanus diphtheria and acellular pertussis (Tdap) immunization during pregnancy in mothers and infants: a randomized clinical trial. JAMA. 2014; 311(17): 1760-9. doi: 10.1001/jama.2014.3633.

16.- Ministerio de Salud Pública. Estadísticas Vitales. Nacimientos. Consultado septiembre 2020. Disponible en: https://uins.msp.gub.uy/.

17.- Recomendaciones de la OMS sobre atención prenatal para una experiencia positiva del embarazo. Washington, D.C. Organización Panamericana de la Salud; 2018. https://iris.paho.org/bitstream/ handle/10665.2/49550/9789275320334_spa. pdf?ua $=1$.

18.- Pertussis vaccination programme for pregnant women update: coverage (England), Jan-March 2019. Health Protection Report. Volume 13 Number 26. Disponible en: https://assets.publishing.service.gov. uk/government/uploads/system/uploads/ attachment data/file/821145/hpr2619 prntlprtsss_VC.pdf.

19.- Kahn E, Black C L, Ding H, Williams W W, Lu P J, Fiebelkorn A P, et al. Influenza and Tdap vaccination coverage among pregnant women-United States, April 2018. MMWR Morbid Mortal Wkly Rep. 2018; 67 (38): 1055 9. doi: 10.15585/mmwr.mm6738a3.

20.- Lindley M, Kahn K E, Bardenheier B H, D’Angelo D V, Dawood F S, Fink R V, et al. Vital signs: burden and prevention of influenza and pertussis among pregnant women and infants, United States. MMWR Morbid Mortal Wkly Rep. 2019; 68 (40): 885-92. http://dx.doi. org/10.15585/mmwr.mm6840e1.

21.- MacDougall D M, Halperin S A. Improving rates of maternal immunization: Challenges and opportunities. Hum Vaccin Immunother. 2016; 12 (4): 857-65. doi: 10.1080/21645515.2015.1101524. 
22.- Yuen C Y, Tarrant M. Determinants of uptake of influenza vaccination among pregnant women- a systematic review. Vaccine. 2014; 32 (36): 4602-13. doi: 10.1016/j. vaccine.2014.06.067.

23.- Krishnaswamy S, Cheng A C, Wallace E U, Buttery J, Giles M L. Understanding the barriers to uptake of antenatal vaccination by women from culturally and linguistically diverse backgrounds: A cross-sectional study. Human Vaccines Immunother. 2018. 14 (7): 1591-8. doi: 10.1080/21645515.2018.1445455.

24.- Maisa A, Milligan S, Quinn A, Boulter D, Johnston J, Treanor C, et al. Vaccination against pertussis and influenza in pregnancy: a qualitative study of barriers and facilitators.
Public Health. 2018; 162: 111-7. doi: 10.1016/j. puhe.2018.05.025.

25.- Mohammed H, McMillan M, Roberts C T, Marshall H S. A systematic review of interventions to improve uptake of pertussis vaccination in pregnancy. PLoS One. 2019 Mar 28; 14 (3): e0214538. doi: 10.1371/journal. pone. 0214538 . 